\title{
A characterization of linearized polynomials with maximum kernel
}

\author{
Bence Csajbók, Giuseppe Marino, Olga Polverino, Ferdinando Zullo*
}

\begin{abstract}
We provide sufficient and necessary conditions for the coefficients of a $q$-polynomial $f$ over $\mathbb{F}_{q^{n}}$ which ensure that the number of distinct roots of $f$ in $\mathbb{F}_{q^{n}}$ equals the degree of $f$. We say that these polynomials have maximum kernel. As an application we study in detail $q$-polynomials of degree $q^{n-2}$ over $\mathbb{F}_{q^{n}}$ which have maximum kernel and for $n \leq 6$ we list all $q$-polynomials with maximum kernel. We also obtain information on the splitting field of an arbitrary $q$ polynomial. Analogous results are proved for $q^{s}$-polynomials as well, where $\operatorname{gcd}(s, n)=1$.
\end{abstract}

AMS subject classification: 11T06, 15A04

Keywords: Linearized polynomials, linear transformations, semilinear transformations

\section{Introduction}

A $q$-polynomial over $\mathbb{F}_{q^{n}}$ is a polynomial of the form $f(x)=\sum_{i} a_{i} x^{q^{i}}$, where $a_{i} \in \mathbb{F}_{q^{n}}$. We will denote the set of these polynomials by $\mathcal{L}_{n, q}$. Let $\mathbb{K}$ denote

${ }^{*}$ The research was supported by Ministry for Education, University and Research of Italy MIUR (Project PRIN 2012 "Geometrie di Galois e strutture di incidenza") and by the Italian National Group for Algebraic and Geometric Structures and their Applications (GNSAGA - INdAM). The first author was supported by the János Bolyai Research Scholarship of the Hungarian Academy of Sciences and by OTKA Grant No. K 124950. 
the algebraic closure of $\mathbb{F}_{q^{n}}$. Then for every $\mathbb{F}_{q^{n}} \leq \mathbb{L} \leq \mathbb{K}, f$ defines an $\mathbb{F}_{q^{-}}$ linear transformation of $\mathbb{L}$, when $\mathbb{L}$ is viewed as an $\mathbb{F}_{q}$-vector space. If $\mathbb{L}$ is a finite field of size $q^{m}$ then the polynomials of $\mathcal{L}_{n, q}$ considered modulo $\left(x^{q^{m}}-x\right)$ form an $\mathbb{F}_{q}$-subalgebra of the $\mathbb{F}_{q}$-linear transformations of $\mathbb{L}$. Once this field $\mathbb{L}$ is fixed, we can define the kernel of $f$ as the kernel of the corresponding $\mathbb{F}_{q}$-linear transformation of $\mathbb{L}$, which is the same as the set of roots of $f$ in $\mathbb{L}$; and the rank of $f$ as the rank of the corresponding $\mathbb{F}_{q}$-linear transformation of $\mathbb{L}$. Note that the kernel and the rank of $f$ depend on this field $\mathbb{L}$ and from now on we will consider the case $\mathbb{L}=\mathbb{F}_{q^{n}}$. In this case $\mathcal{L}_{n, q}$ considered modulo $\left(x^{q^{n}}-x\right)$ is isomorphic to the $\mathbb{F}_{q}$-algebra of $\mathbb{F}_{q}$-linear transformations of the $n$-dimensional $\mathbb{F}_{q^{-}}$-vector space $\mathbb{F}_{q^{n}}$. The elements of this factor algebra are represented by $\tilde{\mathcal{L}}_{n, q}:=\left\{\sum_{i=0}^{n-1} a_{i} x^{q^{i}}: a_{i} \in \mathbb{F}_{q^{n}}\right\}$. For $f \in \tilde{\mathcal{L}}_{n, q}$ if $\operatorname{deg} f=q^{k}$ then we call $k$ the $q$-degree of $f$. It is clear that in this case the kernel of $f$ has dimension at most $k$ and the rank of $f$ is at least $n-k$.

Let $U=\left\langle u_{1}, u_{2}, \ldots, u_{k}\right\rangle_{\mathbb{F}_{q}}$ be a $k$-dimensional $\mathbb{F}_{q^{-}}$-subspace of $\mathbb{F}_{q^{n}}$. It is well known that, up to a scalar factor, there is a unique $q$-polynomial of $q$-degree $k$, which has kernel $U$. We can get such a polynomial as the determinant of the matrix

$$
\left(\begin{array}{cccc}
x & x^{q} & \cdots & x^{q^{k}} \\
u_{1} & u_{1}^{q} & \cdots & u_{1}^{q^{k}} \\
\vdots & & & \\
u_{k} & u_{k}^{q} & \cdots & u_{k}^{q^{k}}
\end{array}\right) .
$$

The aim of this paper is to study the other direction, i.e. when a given $f \in \tilde{\mathcal{L}}_{n, q}$ with $q$-degree $k$ has kernel of dimension $k$. If this happens then we say that $f$ is a $q$-polynomial with maximum kernel.

If $f(x) \equiv a_{0} x+a_{1} x^{\sigma}+\cdots+a_{k} x^{\sigma^{k}}\left(\bmod x^{q^{n}}-x\right)$, with $\sigma=q^{s}$ for some $s$ with $\operatorname{gcd}(s, n)=1$, then we say that $f(x)$ is a $\sigma$-polynomial (or $q^{s}$ polynomial) with $\sigma$-degree (or $q^{s}$-degree) $k$. Regarding $\sigma$-polynomials the following is known.

Result 1.1. [7, Theorem 5] Let $\mathbb{L}$ be a cyclic extension of a field $\mathbb{F}$ of degree $n$, and suppose that $\sigma$ generates the Galois group of $\mathbb{L}$ over $\mathbb{F}$. Let $k$ be an integer satisfying $1 \leq k \leq n$, and let $a_{0}, a_{1}, \ldots, a_{k}$ be elements of $\mathbb{L}$, not all them are zero. Then the $\mathbb{F}$-linear transformation defined as

$$
f(x)=a_{0} x+a_{1} x^{\sigma}+\cdots+a_{k} x^{\sigma^{k}}
$$

has kernel with dimension at most $k$ in $\mathbb{L}$. 
Similarly to the $s=1$ case we will say that a $\sigma$-polynomial is of maximum kernel if the dimension of its kernel equals its $\sigma$-degree.

Linearized polynomials have been used to describe families of $\mathbb{F}_{q}$-linear

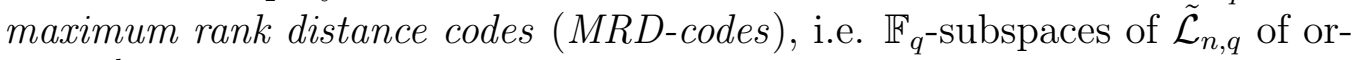
$\operatorname{der} q^{n k}$ in which each element has kernel of dimension at most $k$. The first examples of MRD-codes found were the generalized Gabidulin codes [3, 5], that is $\mathcal{G}_{k, s}=\left\langle x, x^{q^{s}}, \ldots, x^{q^{s(k-1)}}\right\rangle_{\mathbb{F}_{q^{n}}}$ with $\operatorname{gcd}(s, n)=1$; the fact that $\mathcal{G}_{k, s}$ is an MRD-code can be shown simply by using Result 1.1. It is important to have explicit conditions on the coefficients of a linearized polynomial characterizing the number of its roots. Further connections with projective polynomials can be found in [8].

Our main result provides sufficient and necessary conditions on the coefficients of a $\sigma$-polynomial with maximum kernel.

Theorem 1.2. Consider

$$
f(x)=a_{0} x+a_{1} x^{\sigma}+\cdots+a_{k-1} x^{\sigma^{k-1}}-x^{\sigma^{k}},
$$

with $\sigma=q^{s}, \operatorname{gcd}(s, n)=1$ and $a_{0}, \ldots, a_{k-1} \in \mathbb{F}_{q^{n}}$. Then $f(x)$ is of maximum kernel if and only if the matrix

$$
A=\left(\begin{array}{ccccc}
0 & 0 & \cdots & 0 & a_{0} \\
1 & 0 & \cdots & 0 & a_{1} \\
0 & 1 & \cdots & 0 & a_{2} \\
\vdots & \vdots & & \vdots & \vdots \\
0 & 0 & \cdots & 1 & a_{k-1}
\end{array}\right)
$$

satisfies

$$
A A^{\sigma} \cdots A^{\sigma^{n-1}}=I_{k},
$$

where $A^{\sigma^{i}}$ is the matrix obtained from $A$ by applying to each of its entries the automorphism $x \mapsto x^{\sigma^{i}}$ and $I_{k}$ is the identity matrix of order $k$.

An immediate consequence of this result gives information on the splitting field of an arbitrary $\sigma$-polynomial, cf. Theorem 4.1.

In Section 3.1 we study in details the $\sigma$-polynomials of $\sigma$-degree $n-2$ for each $n$. For $n \leq 6$ we also provide a list of all $\sigma$-polynomials with maximum kernel cf. Sections $3.2,3.3$ and 3.4. These results might yield further classification results and examples of $\mathbb{F}_{q}$-linear MRD-codes. 


\section{Preliminary Results}

In this section we recall some results of Dempwolff, Fisher and Herman from [4], adapting them to our needs in order to make this paper self-contained.

Let $V$ be a $k$-dimensional vector space over the field $\mathbb{F}$ and let $T$ be a semilinear transformation of $V$. A $T$-cyclic subspace of $V$ is an $\mathbb{F}$-subspace of $V$ spanned by $\{\mathbf{v}, T(\mathbf{v}), \ldots\}$ over $\mathbb{F}$ for some $\mathbf{v} \in V$, which will be denoted by $[\mathbf{v}]$. We first recall the following lemma.

Lemma 2.1. [4, Theorem 1] Let $V$ be an $n$-dimensional vector space over the field $\mathbb{F}, \sigma$ an automorphism of $\mathbb{F}$ and $T$ an invertible $\sigma$-semilinear transformation on $V$. Then

$$
V=\left[\mathbf{u}_{1}\right] \oplus \ldots \oplus\left[\mathbf{u}_{r}\right]
$$

for $T$-cyclic subspaces satisfying $\operatorname{dim}\left[\mathbf{u}_{1}\right] \geq \operatorname{dim}\left[\mathbf{u}_{2}\right] \geq \ldots \geq \operatorname{dim}\left[\mathbf{u}_{r}\right] \geq 1$.

Theorem 2.2. Let $T$ be an invertible semilinear transformation of $V=$ $V\left(k, q^{n}\right)$ of order $n$, with companion automorphism $\sigma \in \operatorname{Aut}\left(\mathbb{F}_{q^{n}}\right)$ such that $\operatorname{Fix}(\sigma)=\mathbb{F}_{q}$. Then $\operatorname{Fix}(T)$ is a $k$-dimensional $\mathbb{F}_{q^{-}}$-subspace of $V$ and $\langle\operatorname{Fix}(T)\rangle_{\mathbb{F}_{q^{n}}}=$ $V$.

Proof. First assume that the companion automorphism of $T$ is $x \mapsto x^{q}$ and that there exists $\mathbf{v} \in V$ such that

$$
V=\left\langle\mathbf{v}, T(\mathbf{v}), \ldots, T^{k-1}(\mathbf{v})\right\rangle_{\mathbb{F}_{q^{n}}}
$$

Following the proof of [4, Main Theorem], consider the ordered basis $\mathcal{B}_{T}=$ $\left(\mathbf{v}, T(\mathbf{v}), \ldots, T^{k-1}(\mathbf{v})\right)$ and let $A$ be the matrix associated with $T$ with respect to the basis $\mathcal{B}_{T}$, i.e.

$$
A=\left(\begin{array}{ccccc}
0 & 0 & \cdots & 0 & \alpha_{0} \\
1 & 0 & \cdots & 0 & \alpha_{1} \\
0 & 1 & \cdots & 0 & \alpha_{2} \\
\vdots & \vdots & & \vdots & \vdots \\
0 & 0 & \cdots & 1 & \alpha_{k-1}
\end{array}\right) \in \mathbb{F}_{q^{n}}^{k \times k}
$$

where $T^{k}(\mathbf{v})=\sum_{i=1}^{k} \alpha_{i-1} T^{i}(\mathbf{v})$ with $\alpha_{0}, \ldots, \alpha_{k-1} \in \mathbb{F}_{q^{n}}$ and, since $T$ is invertible, we have $\alpha_{0} \neq 0$. Denote by $\bar{T}$ the semilinear transformation of $\mathbb{F}_{q^{n}}^{k}$ having $A$ as the associated matrix with respect to the canonical ordered basis $\mathcal{B}_{C}=\left(\mathbf{e}_{1}, \ldots, \mathbf{e}_{k}\right)$ of $\mathbb{F}_{q^{n}}^{k}$ and companion automorphism $x \mapsto x^{q}$. Note 
that $c_{\mathcal{B}_{T}}(\operatorname{Fix}(T))=\operatorname{Fix}(\bar{T})$, where $c_{\mathcal{B}_{T}}$ is the coordinatization with respect to the basis $\mathcal{B}_{T}$. Also, since $T$ has order $n$, we have

$$
A A^{q} \cdots A^{q^{n-1}}=I_{k}
$$

where $A^{q^{i}}$, for $i \in\{1, \ldots, n-1\}$, is the matrix obtained from $A$ by applying to each of its entries the automorphism $x \mapsto x^{q^{i}}$. A vector $\mathbf{z}=\left(z_{0}, \ldots, z_{k-1}\right) \in$ $\mathbb{F}_{q^{n}}^{k}$ is fixed by $\bar{T}$ if and only if

$$
\left\{\begin{array}{l}
\alpha_{0} z_{k-1}^{q}=z_{0} \\
z_{0}^{q}+\alpha_{1} z_{k-1}^{q}=z_{1} \\
\vdots \\
z_{k-2}^{q}+\alpha_{k-1} z_{k-1}^{q}=z_{k-1}
\end{array}\right.
$$

Eliminating $z_{0}, \ldots, z_{k-2}$, we obatin the equation

$$
\alpha_{0}^{q^{k-1}} z_{k-1}^{q^{k}}+\alpha_{1}^{q^{k-2}} z_{k-1}^{q^{k-1}}+\ldots+\alpha_{k-1} z_{k-1}^{q}-z_{k-1}=0,
$$

which has $q^{k}$ distinct solutions in the algebraic closure $\mathbb{K}$ of $\mathbb{F}_{q^{n}}$ by the derivative test. Each solution determines a unique vector of $\operatorname{Fix}(\bar{T})$ in $\mathbb{K}^{k}$. Also,

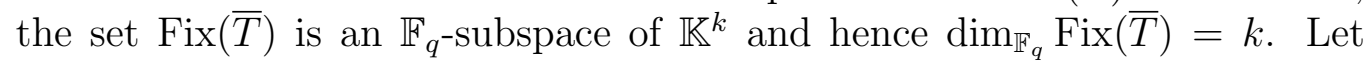
$\left\{\mathbf{w}_{1}, \ldots, \mathbf{w}_{k}\right\}$ be an $\mathbb{F}_{q}$-basis of $\operatorname{Fix}(\bar{T})$ and note that since $|\operatorname{Fix}(\bar{T})|=q^{k}$, a vector $\sum_{i=1}^{k} a_{i} \mathbf{w}_{i}$ is fixed by $\bar{T}$ if and only if $a_{i} \in \mathbb{F}_{q}$. This implies that $\mathbf{w}_{1}, \ldots, \mathbf{w}_{k}$ are also $\mathbb{K}$-independent. Thus $\langle\operatorname{Fix}(\bar{T})\rangle_{\mathbb{K}}=\mathbb{K}^{k}$ and $\left\{\mathbf{w}_{1}, \ldots, \mathbf{w}_{k}\right\}$ is also a $\mathbb{K}$-basis of $\mathbb{K}^{k}$. Denote by $\phi$ the $\mathbb{K}$-linear transformation such that $\phi\left(\mathbf{w}_{i}\right)=\mathbf{e}_{i}$ and by $P$ the associated matrix with respect to the canonical basis $\mathcal{B}_{C}$, so $P \in \mathrm{GL}(k, \mathbb{K})$. The semilinear transformation $\phi \circ \bar{T} \circ \phi^{-1}$ has companion automorphism $x \mapsto x^{q}$, order $n$ and associated matrix with respect to the canonical basis $P \cdot A \cdot P^{-q}$, where $P^{-q}$ is the inverse of $P$ in which the automorphism $x \mapsto x^{q}$ is applied entrywise. Note that $\phi \circ \bar{T} \circ \phi^{-1}\left(\mathbf{e}_{i}\right)=\phi\left(\bar{T}\left(\mathbf{w}_{i}\right)\right)=\phi\left(\mathbf{w}_{i}\right)=\mathbf{e}_{i}$, hence

$$
P \cdot A \cdot P^{-q}=I_{k}
$$

i.e.

$$
P^{q}=P \cdot A
$$

By Equations (3) and (5) and using induction we get

$$
P^{q^{n}}=P \cdot A \cdot A^{q} \cdot \ldots \cdot A^{q^{n-1}}=P
$$




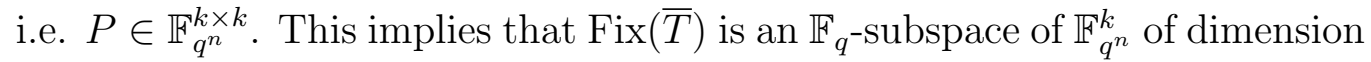
$k$ and hence $\operatorname{Fix}(T)=c_{\mathcal{B}_{T}}^{-1}(\operatorname{Fix}(\bar{T}))$ is a $k$-dimensional subspace of $V\left(k, q^{n}\right)$ with the property that $\langle\operatorname{Fix}(T)\rangle_{\mathbb{F}_{q^{n}}}=V$.

Consider now the general case, i.e. suppose $T$ as in the statement, that is $T$ is an invertible semilinear map of order $n$ with companion automorphism $x \mapsto x^{q^{s}}$ and $\operatorname{gcd}(s, n)=1$. Since $\operatorname{gcd}(s, n)=1$ there exist $l, m \in \mathbb{N}$ such that $1=s l+m n$, and hence $\operatorname{gcd}(l, n)=1$. Then the semilinear transformation $T^{l}$ has order $n$, companion automorphism $x \mapsto x^{q}$ and $\operatorname{Fix}(T)=\operatorname{Fix}\left(T^{l}\right)$. By Lemma 2.1, we may write

$$
V=\left[\mathbf{u}_{1}\right] \oplus \ldots \oplus\left[\mathbf{u}_{r}\right]
$$

where $\left[\mathbf{u}_{i}\right]$ is a $T^{l}$-cyclic subspace of $V$ of dimension $m_{i} \geq 1$, for each $i \in$ $\{1, \ldots, r\}$, and $\sum_{i=1}^{r} m_{i}=k$. Then we can restrict $T^{l}$ to each subspace $\left[\mathbf{u}_{i}\right]$ and by applying the previous arguments we get that $U_{i}=\operatorname{Fix}\left(\left.T^{l}\right|_{\left[\mathbf{u}_{i}\right]}\right)$ is an $\mathbb{F}_{q^{-}}$-subspace of $\left[\mathbf{u}_{i}\right]$ of dimension $m_{i}$ with the property that $\left\langle U_{i}\right\rangle_{\mathbb{F}_{q^{n}}}=\left[\mathbf{u}_{i}\right]$. Thus

$$
\operatorname{Fix}(T)=\operatorname{Fix}\left(T^{l}\right)=U_{1} \oplus \ldots \oplus U_{r}
$$

is an $\mathbb{F}_{q^{-}}$-subspace of dimension $k$ of $V$ with the property that $\langle\operatorname{Fix}(T)\rangle_{\mathbb{F}_{q^{n}}}=$ $V$.

The existence of a matrix $P \in \mathrm{GL}(k, \mathbb{K})$, with $\mathbb{K}$ the algebraic closure of a finite field of order $q$, satisfying (4) is also a consequence of the celebrated Lang's Theorem [9] on connected linear algebraic groups. More precisely, by Lang's Theorem, since $\mathrm{GL}(k, \mathbb{K})$ is a connected linear algebraic group, the map $M \in \mathrm{GL}(k, \mathbb{K}) \mapsto M^{-1} \cdot M^{q} \in \mathrm{GL}(k, \mathbb{K})$ is onto. In Theorem 2.2 it is proved that, if the semilinear transformation of $V\left(k, q^{n}\right)$ having $A$ as associated matrix has order $n$, then $P \in \mathrm{GL}\left(k, \mathbb{F}_{q^{n}}\right)$.

Remark 2.3. Let $T$ be an invertible semilinear transformation of $V=$ $V\left(k, q^{n}\right)$ with companion automorphism $x \mapsto x^{q}$ and let $\mathbb{K}$ be the algebraic closure of $\mathbb{F}_{q^{n}}$. Denote by $\bar{T}$ the semilinear transformation of $\mathbb{K}^{k}$ associated with $T$ as in the proof of Theorem 2.2. If $\lambda \in \mathbb{K}$, then the set $E(\lambda):=\left\{\mathbf{v} \in \mathbb{K}^{k}: \bar{T}(\mathbf{v})=\lambda \mathbf{v}\right\}$ is an $\mathbb{F}_{q^{-}}$subspace of $\mathbb{K}^{k}$. By [4, page 293], it follows that $E(\lambda)=\lambda^{\frac{1}{q-1}} \operatorname{Fix}(\bar{T})$ and by [4, Main Theorem] $E(\lambda)$ is a $k$ dimensional $\mathbb{F}_{q^{-}}$subspace of $\mathbb{K}^{k}$. Also, when $T$ has order $n$ and $\lambda^{\frac{1}{q-1}} \in \mathbb{F}_{q^{n}}$, by Theorem 2.2. $E(\lambda)$ is a $k$-dimensional $\mathbb{F}_{q^{-}}$-subspace contained in $\mathbb{F}_{q^{n}}^{k}$ such that $\langle E(\lambda)\rangle_{\mathbb{F}_{q^{n}}}=\mathbb{F}_{q^{n}}^{k}$. 


\section{Main Results}

Now we are able to prove our main result:

Proof of Theorem 1.2. First suppose $\operatorname{dim}_{\mathbb{F}_{q}} \operatorname{ker} f=k$. Then there exist $u_{0}, u_{1}, \ldots, u_{k-1} \in \mathbb{F}_{q^{n}}$ which form an $\mathbb{F}_{q^{-}}$basis of ker $f$.

Put $\mathbf{u}:=\left(u_{0}, u_{1}, \ldots, u_{k-1}\right) \in \mathbb{F}_{q^{n}}^{k}$. Since $u_{0}, u_{1}, \ldots, u_{k-1}$ are $\mathbb{F}_{q}$-linearly independent, by [10, Lemma 3.51], we get that $\mathcal{B}:=\left(\mathbf{u}, \mathbf{u}^{q^{s}}, \ldots, \mathbf{u}^{q^{s(k-1)}}\right)$ is an

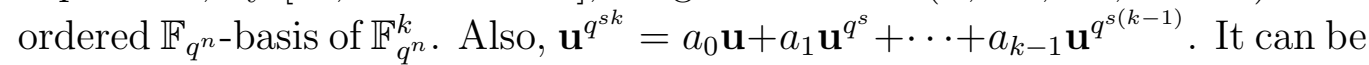
seen that the matrix (1) represents the $\mathbb{F}_{q^{n}}$-linear part of the $\mathbb{F}_{q^{n} \text {-semilinear }}$ map $\bar{\sigma}: \mathbf{v} \in \mathbb{F}_{q^{n}}^{k} \mapsto \mathbf{v}^{q^{s}} \in \mathbb{F}_{q^{n}}^{k}$ w.r.t. the basis $\mathcal{B}$. Since $\operatorname{gcd}(s, n)=1, \bar{\sigma}$ has order $n$ and hence the assertion follows.

Viceversa, let $\tau$ be defined as follows

$$
\tau:\left(\begin{array}{c}
x_{0} \\
x_{1} \\
\vdots \\
x_{k-1}
\end{array}\right) \in \mathbb{F}_{q^{n}}^{k} \mapsto A\left(\begin{array}{c}
x_{0} \\
x_{1} \\
\vdots \\
x_{k-1}
\end{array}\right)^{q^{s}} \in \mathbb{F}_{q^{n}}^{k}
$$

where $A$ is as in (1) with the property $A A^{q^{s}} \cdots A^{q^{s(n-1)}}=I_{k}$. Then $\tau$ has

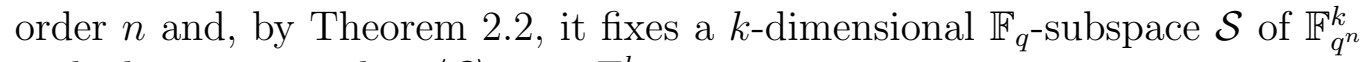
with the property that $\langle\mathcal{S}\rangle_{\mathbb{F}_{q^{n}}}=\mathbb{F}_{q^{n}}^{k}$.

Let $\mathcal{B}_{\mathcal{S}}=\left(\mathbf{s}_{0}, \ldots, \mathbf{s}_{k-1}\right)$ be an $\mathbb{F}_{q^{-}}$-basis of $\mathcal{S}$ and note that, since $\langle\mathcal{S}\rangle_{\mathbb{F}_{q^{n}}}=$

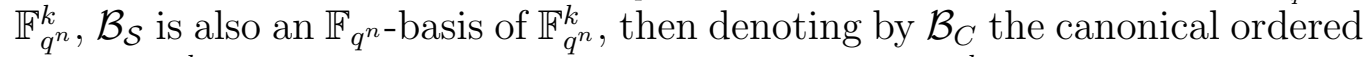
basis of $\mathbb{F}_{q^{n}}^{k}$, there exists a unique isomorphism $\phi$ of $\mathbb{F}_{q^{n}}^{k}$ such that $\phi\left(\mathbf{s}_{i}\right)=\mathbf{e}_{i}$ for each $i \in\{1, \ldots, k\}$. Then $\bar{\sigma}=\phi \circ \tau \circ \phi^{-1}$, where $\bar{\sigma}: \mathbf{v} \in \mathbb{F}_{q^{n}}^{k} \mapsto \mathbf{v}^{q^{s}} \in \mathbb{F}_{q^{n}}^{k}$. Also,

$$
\bar{\sigma}^{i}=\phi \circ \tau^{i} \circ \phi^{-1}
$$

for each $i \in\{1, \ldots, n-1\}$. Also, by (6)

$$
\begin{aligned}
& \tau\left(\mathbf{e}_{0}\right)=\mathbf{e}_{1}, \\
& \tau\left(\mathbf{e}_{1}\right)=\tau^{2}\left(\mathbf{e}_{0}\right)=\mathbf{e}_{2}, \\
& \vdots \\
& \tau\left(\mathbf{e}_{k-1}\right)=\tau^{k}\left(\mathbf{e}_{0}\right)=\left(a_{0}, \ldots, a_{k-1}\right)=a_{0} \mathbf{e}_{0}+\cdots+a_{k-1} \mathbf{e}_{k-1} .
\end{aligned}
$$

So, we get that

$$
\tau^{k}\left(\mathbf{e}_{0}\right)=a_{0} \mathbf{e}_{0}+a_{1} \tau\left(\mathbf{e}_{0}\right)+\cdots+a_{k-1} \tau^{k-1}\left(\mathbf{e}_{0}\right)
$$


and applying $\phi$ it follows that

$$
\phi\left(\tau^{k}\left(\mathbf{e}_{0}\right)\right)=a_{0} \phi\left(\mathbf{e}_{0}\right)+a_{1} \phi\left(\tau\left(\mathbf{e}_{0}\right)\right)+\cdots+a_{k-1} \phi\left(\tau^{k-1}\left(\mathbf{e}_{0}\right)\right) .
$$

By (7) the previous equation becomes

$$
\bar{\sigma}^{k}\left(\phi\left(\mathbf{e}_{0}\right)\right)=a_{0} \phi\left(\mathbf{e}_{0}\right)+a_{1} \bar{\sigma}\left(\phi\left(\mathbf{e}_{0}\right)\right)+\cdots+a_{k-1} \bar{\sigma}^{k-1}\left(\phi\left(\mathbf{e}_{0}\right)\right) .
$$

Put $\mathbf{u}=\phi\left(\mathbf{e}_{0}\right)$, then

$$
\mathbf{u}^{q^{s k}}=a_{0} \mathbf{u}+a_{1} \mathbf{u}^{q^{s}}+\cdots+a_{k-1} \mathbf{u}^{q^{s(k-1)}} .
$$

This implies that $u_{0}, u_{1}, \ldots, u_{k-1}$ are elements of ker $f$, where $\mathbf{u}=\left(u_{0}, \ldots, u_{k-1}\right)$. Also, they are $\mathbb{F}_{q^{-}}$independent since $\mathcal{B}=\left(\mathbf{u}, \ldots, \mathbf{u}^{q^{s(k-1)}}\right)=$ $\left(\phi\left(\mathbf{e}_{0}\right), \ldots, \phi\left(\mathbf{e}_{k-1}\right)\right)$ is an ordered $\mathbb{F}_{q^{n}}$-basis of $\mathbb{F}_{q^{n}}^{k}$. This completes the proof.

As a corollary we get the second part of [6, Theorem 10], see also [12, Lemma 3] for the case $s=1$ and [11] for the case when $q$ is a prime. Indeed, by evaluating the determinants in $A A^{q^{s}} \cdots A^{q^{s(n-1)}}=I_{k}$ we obtain the following corollary $!^{1}$

Corollary 3.1. If the kernel of a $q^{s}$-polynomial $f(x)=a_{0} x+a_{1} x^{q^{s}}+\cdots+$ $a_{k-1} x^{q^{s(k-1)}}-x^{q^{s k}}$ has dimension $k$, then $\mathrm{N}\left(a_{0}\right)=(-1)^{n(k+1)}$.

Corollary 3.2. Let $A$ be a matrix as in Theorem 1.2. The condition

$$
A A^{q^{s}} \cdots A^{q^{s(n-1)}}=I_{k}
$$

is satisfied if and only if $A A^{q^{s}} \cdots A^{q^{s(n-1)}}$ fixes $\mathbf{e}_{0}=(1,0, \ldots, 0)$.

Proof. The only if part is trivial, we prove the if part by induction on $0 \leq$ $i \leq k-1$. Suppose $A A^{q^{s}} \cdots A^{q^{s(n-1)}} \mathbf{e}_{i}^{T}=\mathbf{e}_{i}^{T}$ for some $0 \leq i \leq k-1$. Then by taking $q^{s}$-th powers of each entry we get $A^{q^{s}} A^{q^{2 s}} \cdots A \mathbf{e}_{i}^{T}=\mathbf{e}_{i}^{T}$. Since $A \mathbf{e}_{i}^{T}=\mathbf{e}_{i+1}^{T}$ this yields $A^{q^{s}} A^{q^{2 s}} \cdots A^{q^{s(n-1)}} \mathbf{e}_{i+1}^{T}=\mathbf{e}_{i}^{T}$. Then multiplying both sides by $A$ yields $A A^{q^{s}} A^{q^{2 s}} \cdots A^{q^{s(n-1)}} \mathbf{e}_{i+1}^{T}=\mathbf{e}_{i+1}^{T}$.

\footnotetext{
${ }^{1}$ For $x \in \mathbb{F}_{q^{n}}$ and for a subfield $\mathbb{F}_{q^{m}}$ of $\mathbb{F}_{q^{n}}$ we will denote by $\mathrm{N}_{q^{n} / q^{m}}(x)$ the norm of $x$ over $\mathbb{F}_{q^{m}}$ and by $\operatorname{Tr}_{q^{n} / q^{m}}(x)$ we will denote the trace of $x$ over $\mathbb{F}_{q^{m}}$. If $n$ is clear from the context and $m=1$ then we will simply write $\mathrm{N}(x)$ and $\operatorname{Tr}(x)$.
} 
Consider a $q^{s}$-polynomial $f(x)=a_{0} x+a_{1} x^{q^{s}}+\cdots+a_{k-1} x^{q^{s(k-1)}}-x^{q^{s k}}$, the matrix $A \in \mathbb{F}_{q^{n}}^{k \times k}$ as in Theorem 1.2 and the semilinear map $\tau$ defined in (6).

Note that

$$
\begin{gathered}
\mathbf{e}_{0}^{\tau}=(0,1,0, \ldots, 0)=\mathbf{e}_{1} \\
\mathbf{e}_{0}^{\tau^{2}}=(0,0,1, \ldots, 0)=\mathbf{e}_{2} \\
\vdots \\
\mathbf{e}_{0}^{\tau^{k-1}}=(0,0,0, \ldots, 1)=\mathbf{e}_{k-1} \\
\mathbf{e}_{0}^{\tau^{k}}=\left(a_{0}, a_{1}, a_{2}, \ldots, a_{k-1}\right) \\
\mathbf{e}_{0}^{\tau^{k+1}}=\left(a_{0} a_{k-1}^{q^{s}}, a_{0}^{q^{s}}+a_{1} a_{k-1}^{q^{s}}, a_{1}^{q^{s}}+a_{2} a_{k-1}^{q^{s}}, \ldots, a_{k-2}^{q^{s}}+a_{k-1}^{q^{s}+1}\right) .
\end{gathered}
$$

Hence, if

$$
\mathbf{e}_{0}^{\tau^{i}}=\left(Q_{0, i}, Q_{1, i}, \ldots, Q_{k-1, i}\right)
$$

where $Q_{j, i}$ can be seen as polynomials in $a_{0}, a_{1}, \ldots, a_{k-1}$, for $i \geq 0$, then

$$
\mathbf{e}_{0}^{\tau^{i+1}}=\left(a_{0} Q_{k-1, i}^{q^{s}}, Q_{0, i}^{q^{s}}+a_{1} Q_{k-1, i}^{q^{s}}, \ldots, Q_{k-2, i}^{q^{s}}+a_{k-1} Q_{k-1, i}^{q^{s}}\right),
$$

i.e. the polynomials $Q_{j, i}$ for $0 \leq j \leq k-1$ can be defined by the following recursive relations for $0 \leq i \leq k-1$ :

$$
Q_{j, i}= \begin{cases}1 & \text { if } j=i \\ 0 & \text { otherwise }\end{cases}
$$

and by the following relations for $i \geq k$ :

$$
\begin{aligned}
& Q_{0, i+1}=a_{0} Q_{k-1, i}^{q^{s}} \\
& Q_{j, i+1}=Q_{j-1, i}^{q^{s}}+a_{j} Q_{k-1, i}^{q^{s}}
\end{aligned}
$$

Now, we are able to prove the following.

Theorem 3.3. The kernel of a $q^{s}$-polynomial $f(x)=a_{0} x+a_{1} x^{q^{s}}+\cdots+$ $a_{k-1} x^{q^{s(k-1)}}-x^{q^{s k}} \in \mathbb{F}_{q^{n}}[x]$, where $\operatorname{gcd}(s, n)=1$, has dimension $k$ if and only if

$$
Q_{j, n}\left(a_{0}, a_{1}, \ldots, a_{k-1}\right)= \begin{cases}1 & \text { if } j=0 \\ 0 & \text { otherwise }\end{cases}
$$


Proof. Relations (9) can be written as follows

$$
\left(\begin{array}{c}
Q_{0, i+1} \\
Q_{1, i+1} \\
\vdots \\
Q_{k-1, i+1}
\end{array}\right)=\left(\begin{array}{ccccc}
0 & 0 & \cdots & 0 & a_{0} \\
1 & 0 & \cdots & 0 & a_{1} \\
0 & 1 & \cdots & 0 & a_{2} \\
\vdots & \vdots & & \vdots & \vdots \\
0 & 0 & \cdots & 1 & a_{k-1}
\end{array}\right)\left(\begin{array}{c}
Q_{0, i}^{q^{s}} \\
Q_{1, i}^{q^{s}} \\
\vdots \\
Q_{k-1, i}^{q^{s}}
\end{array}\right)
$$

with $i \in\{0, \ldots, n-1\}$. Also, $\left(Q_{0,0}, Q_{1,0}, \ldots, Q_{k-1,0}\right)=(1,0, \ldots, 0)$ and $\mathbf{e}_{0}^{\tau^{t}}=$ $\left(Q_{0, t}, \ldots, Q_{k-1, t}\right)$ for $t \in\{0, \ldots, n\}$. By Theorem 1.2 and by Corollary 3.2, the kernel of $f(x)$ has dimension $k$ if and only if $\mathbf{e}_{0}=\left(Q_{0,0}, Q_{1,0}, \ldots, Q_{k-1,0}\right)$ is fixed by $A A^{q^{s}} \cdots A^{q^{s(n-1)}}$, so this happens if and only if

$$
\mathbf{e}_{0}^{\tau^{n}}=\left(Q_{0, n}, Q_{1, n}, \ldots, Q_{k-1, n}\right)=(1,0, \ldots, 0) .
$$

Theorem 3.3 with $k=n-1$ and $s=1$ gives the following well-known result as a corollary.

Corollary 3.4. [10, Theorem 2.24] The dimension of the kernel of a $q$ polynomial $f(x) \in \mathbb{F}_{q^{n}}[x]$ is $n-1$ if and only if there exist $\alpha, \beta \in \mathbb{F}_{q^{n}}^{*}$ such that

$$
f(x)=\alpha \operatorname{Tr}(\beta x) .
$$

Again from Theorem 3.3 we can deduce the following.

Corollary 3.5. [10, Ex. 2.14] The $q^{s}$-polynomial $a_{0} x-x^{q^{s k}} \in \mathbb{F}_{q^{n}}[x]$, with $\operatorname{gcd}(s, n)=1$ and $1 \leq k \leq n-1$, admits $q^{k}$ roots if and only if $k \mid n$ and $\mathrm{N}_{q^{n} / q^{k}}\left(a_{0}\right)=1$.

\subsection{When the $q^{s}$-degree equals $n-2$}

In this section we investigate $q^{s}$-polynomials

$$
f(x)=a_{0} x+a_{1} x^{q^{s}}+\cdots+a_{n-3} x^{q^{s(n-3)}}-x^{q^{s(n-2)}}
$$

with $\operatorname{gcd}(s, n)=1$. By Theorem 3.3, $\operatorname{dim} \operatorname{ker} f(x)=n-2$ if and only if $a_{0}, a_{1}, \ldots, a_{n-3}$ satisfy the following system of equations 


$$
\left\{\begin{array}{l}
Q_{0, n}=a_{0}\left(a_{n-4}^{q^{2 s}}+a_{n-3}^{q^{2 s}+q^{s}}\right)=1 \\
Q_{1, n}=a_{0}^{q^{s}} a_{n-3}^{q^{2 s}}+a_{1}\left(a_{n-4}^{q^{2 s}}+a_{n-3}^{q^{2 s}+q^{s}}\right)=0 \\
Q_{2, n}=a_{0}^{q^{2 s}}+a_{n-3}^{q^{2 s}} a_{1}^{q^{s}}+a_{2}\left(a_{n-4}^{q^{2 s}}+a_{n-3}^{q^{2 s}+q^{s}}\right)=0 \\
Q_{3, n}=a_{1}^{q^{2 s}}+a_{n-3}^{q^{2 s}} a_{2}^{q^{s}}+a_{3}\left(a_{n-4}^{q^{2 s}}+a_{n-3}^{q^{2 s}+q^{s}}\right)=0 \\
\vdots \\
Q_{n-3, n}=a_{n-5}^{q^{2 s}}+a_{n-3}^{q^{2 s}} a_{n-4}^{q^{s}}+a_{n-3}\left(a_{n-4}^{q^{2 s}}+a_{n-3}^{q^{2 s}+q^{s}}\right)=0
\end{array}\right.
$$

which is equivalent to

$$
\left\{\begin{array}{l}
a_{0}\left(a_{n-4}^{q^{2 s}}+a_{n-3}^{q^{2 s}+q^{s}}\right)=1 \\
a_{1}=-a_{0}^{q^{s}+1} a_{n-3}^{q^{2 s}}=: g_{1}\left(a_{0}, a_{n-3}\right) \\
a_{j}=-a_{j-2}^{q^{2 s}} a_{0}-a_{n-3}^{q^{2 s}} a_{j-1}^{q^{s}} a_{0}=: g_{j}\left(a_{0}, a_{n-3}\right), \text { for } 2 \leq j \leq n-3
\end{array}\right.
$$

So, $\operatorname{dim}_{\mathbb{F}_{q}} \operatorname{ker} f(x)=n-2$ if and only if $a_{0}$ and $a_{n-3}$ satisfy the equations

$$
\left\{\begin{array}{l}
a_{0}\left(g_{n-4}\left(a_{0}, a_{n-3}\right)^{q^{2 s}}+a_{n-3}^{q^{2 s}+q^{s}}\right)=1 \\
a_{n-3}=g_{n-3}\left(a_{0}, a_{n-3}\right)
\end{array}\right.
$$

and $a_{j}=g_{j}\left(a_{0}, a_{n-3}\right)$ for $j \in\{1, \ldots, n-4\}$.

Theorem 3.6. Suppose that $f(x)=a_{0} x+a_{1} x^{q}+\cdots+a_{n-3} x^{q^{n-3}}-x^{q^{n-2}}$ has maximum kernel. Then for $t \geq 2$ with $\operatorname{gcd}(t-1, n)=1$ the coefficients $a_{t-2}$ and $a_{n-t}$ are non-zero and, with $s=n-t+1$,

$$
a_{n-2 t+1} a_{t-2}^{q^{2 s}+q^{s}}=-a_{n-t}^{q^{s}+1} a_{2 t-3}^{q^{2 s}} .
$$

Also, it holds that

$$
-a_{n-t}\left(-a_{t-2}^{q^{s}} a_{3 t-4}^{q^{2 s}}+a_{2 t-3}^{q^{2 s}+q^{s}}\right)=a_{t-2}^{q^{2 s}+q^{s}+1}
$$

In particular, for $t \geq 2$ with $\operatorname{gcd}(t-1, n)=1$ we get

$$
\mathrm{N}\left(a_{n-t}\right)=(-1)^{n} \mathrm{~N}\left(a_{t-2}\right)
$$

and

$$
\mathrm{N}\left(a_{n-2 t+1}\right)=(-1)^{n} \mathrm{~N}\left(a_{2 t-3}\right),
$$

where $n-2 t+1$ and $2 t-3$ are considered modulo $n$. 
Proof. Let $t \geq 2$ with $\operatorname{gcd}(t-1, n)=1$ and consider the polynomial $F(x)=$ $f\left(x^{q^{t}}\right)$, that is,

$$
F(x)=a_{0} x^{q^{t}}+a_{1} x^{q^{t+1}}+\cdots+a_{n-3} x^{q^{n+t-3}}-x^{q^{n+t-2}} .
$$

Clearly $\operatorname{dim}_{\mathbb{F}_{q}} \operatorname{ker} F=\operatorname{dim}_{\mathbb{F}_{q}} \operatorname{ker} f=n-2$. By renaming the coefficients, $F(x)$ can be written as

$$
\begin{gathered}
F(x)=\alpha_{0} x+\alpha_{1} x^{q^{n-t+1}}+\alpha_{2} x^{q^{2(n-t+1)}}+\cdots+\alpha_{n-3} x^{q^{(n-t+1)(n-3)}}+\alpha_{n-2} x^{q^{(n-t+1)(n-2)}} \\
=\alpha_{0} x+\alpha_{1} x^{q^{n-t+1}}+\cdots+\alpha_{n-3} x^{q^{3 t-3}}+\alpha_{n-2} x^{q^{2 t-2}} .
\end{gathered}
$$

Since $F(x)$ has maximum kernel, by the second equation of 12 we get $\alpha_{0} \neq 0, \alpha_{n-2} \neq 0$ and the following relation

$$
-\frac{\alpha_{1}}{\alpha_{n-2}}=-\left(-\frac{\alpha_{0}}{\alpha_{n-2}}\right)^{q^{s}+1}\left(-\frac{\alpha_{n-3}}{\alpha_{n-2}}\right)^{q^{2 s}} .
$$

The coefficient $\alpha_{j}$ of $F(x)$ equals the coefficient $a_{i}$ of $f(x)$ with $i \equiv n-t+$ $j(1-t)(\bmod n)$, in particular

$$
\left\{\begin{array}{l}
\alpha_{0}=a_{n-t}, \\
\alpha_{1}=a_{n-2 t+1}, \\
\alpha_{n-3}=a_{2 t-3}, \\
\alpha_{n-2}=a_{t-2}, \\
\alpha_{n-4}=a_{3 t-4},
\end{array}\right.
$$

and by (17), we get that $a_{t-2}$ and $a_{n-t}$ are nonzero, and

$$
a_{n-2 t+1} a_{t-2}^{q^{2 s}+q^{s}}=-a_{n-t}^{q^{s}+1} a_{2 t-3}^{q^{2 s}},
$$

which gives (13). The first equation of $(12)$ gives

$$
-\frac{\alpha_{0}}{\alpha_{n-2}}\left(\left(-\frac{\alpha_{n-4}}{\alpha_{n-2}}\right)^{q^{2 s}}+\left(-\frac{\alpha_{n-3}}{\alpha_{n-2}}\right)^{q^{2 s}+q^{s}}\right)=1,
$$

that is,

$$
-\alpha_{0}\left(-\alpha_{n-2}^{q^{s}} \alpha_{n-4}^{q^{2 s}}+\alpha_{n-3}^{q^{2 s}+q^{s}}\right)=\alpha_{n-2}^{q^{2 s}+q^{s}+1} .
$$

Then (18) and $\alpha_{n-4}=a_{3 t-4}$ imply

$$
-a_{n-t}\left(-a_{t-2}^{q^{s}} a_{3 t-4}^{q^{2 s}}+a_{2 t-3}^{q^{2 s}+q^{s}}\right)=a_{t-2}^{q^{2 s}+q^{s}+1},
$$


which gives (14). By Corollary 3.1 with $s=n-t+1$ we obtain

$$
\mathrm{N}\left(-\frac{\alpha_{0}}{\alpha_{n-2}}\right)=1
$$

and taking (18) into account we get

$$
\mathrm{N}\left(a_{n-t}\right)=(-1)^{n} \mathrm{~N}\left(a_{t-2}\right) .
$$

Then (13) and the previous relation yield

$$
\mathrm{N}\left(a_{n-2 t+1}\right)=(-1)^{n} \mathrm{~N}\left(a_{2 t-3}\right) .
$$

Proposition 3.7. Let $f(x)$ be a $q^{s}$-polynomial with $q^{s}$-degree $n-2$ and with maximum kernel. If the coefficient of $x^{q^{s}}$ is zero, then $n$ is even and $f(x)=$ $\alpha \operatorname{Tr}_{q^{n} / q^{2}}(\beta x)$ for some $\alpha, \beta \in \mathbb{F}_{q^{n}}^{*}$.

Proof. We may assume $f(x)=a_{0} x+a_{1} x^{q^{s}}+\cdots+a_{n-3} x^{q^{s(n-3)}}-x^{q^{s(n-2)}}$ with $a_{1}=0$. By the second equation of $(12)$, it follows that $a_{n-3}=0$. By the third equation of $(12)$, we get that $a_{j}=0$ for every odd integer $j \in\{3, \ldots, n-3\}$. If $j$ is even then we have

$$
a_{j}=(-1)^{\frac{j}{2}} a_{0}^{q^{s j}+q^{s(j-2)}+\cdots+q^{2 s}+1} .
$$

If $n-3$ is even, then this gives us a contradiction with $j=n-3$. It follows that $n-3$ is odd and hence $n$ is even. By $\mathrm{N}\left(a_{0}\right)=(-1)^{n}$, there exists $\lambda \in \mathbb{F}_{q^{n}}^{*}$ such that $a_{0}=-\lambda^{1-q^{s(n-2)}}$. So, by 19 we get $a_{j}=\lambda^{q^{j s}-q^{s(n-2)}}$, and hence

$$
f(x)=\frac{\operatorname{Tr}_{q^{n} / q^{2}}(\lambda x)}{\lambda^{q^{s(n-2)}}} .
$$

In the next sections we list all the $q^{s}$-polynomials of $\mathbb{F}_{q^{n}}$ with maximum kernel for $n \leq 6$. By Corollaries 3.4 and 3.5 the $n \leq 3$ case can be easily described hence we will consider only the $n \in\{4,5,6\}$ cases.

For $f(x)=\sum_{i=0}^{n-1} a_{i} x^{q^{i}} \in \tilde{\mathcal{L}}_{n, q}$ we denote by $\hat{f}(x):=\sum_{i=0}^{n-1} a_{i}^{q^{n-i}} x^{q^{n-i}}$ the adjoint (w.r.t. the symmetric non-degenerate bilinear form defined by $\langle x, y\rangle=\operatorname{Tr}(x y))$ of $f$.

By [1, Lemma 2.6], see also [2, pages 407-408], the kernel of $f$ and $\hat{f}$ has the same dimension and hence the following result holds. 
Proposition 3.8. If $f(x) \in \tilde{\mathcal{L}}_{n, q}$ is a $q^{s}$-polynomial with maximum kernel, then $\hat{f}(x)$ is a $q^{n-s}$-polynomial with maximum kernel.

This will allow us to consider only the $s \leq n / 2$ case.

\subsection{The $n=4$ case}

In this section we determine the linearized polynomials over $\mathbb{F}_{q^{4}}$ with maximum kernel. Without loss of generality, we can suppose that the leading coefficient of the polynomial is -1 .

Because of Proposition 3.8, we can assume $s=1$. Corollaries 3.4 and 3.5 cover the cases when the $q$-degree of $f$ is 1 or 3 so from now on we suppose $f(x)=a_{0} x+a_{1} x^{q}-x^{q^{2}}$. If $a_{1}=0$ then we can use again Corollary 3.5 and we get $a_{0} x-x^{q^{2}}$, with $\mathrm{N}_{q^{4} / q^{2}}\left(a_{0}\right)=1$. Suppose $a_{1} \neq 0$. By Equation (12), we get the conditions

$$
\left\{\begin{array}{l}
a_{0}\left(a_{0}^{q^{2}}+a_{1}^{q^{2}+q}\right)=1, \\
a_{1}=-a_{0}^{q+1} a_{1}^{q^{2}},
\end{array}\right.
$$

which is equivalent to

$$
\left\{\begin{array}{l}
\mathrm{N}_{q^{4} / q}\left(a_{0}\right)=1, \\
a_{1}^{q+1}=a_{0}^{q^{2}+q+1}-a_{0}^{q},
\end{array}\right.
$$

see (A1) of Section 5 .

Here we list the $q$-polynomials of $\mathcal{L}_{4, q}$ with maximum kernel, up to a non-zero scalar in $\mathbb{F}_{q^{4}}^{*}$. Applying the adjoint operation we can obtain the list of $q^{3}$-polynomials over $\mathbb{F}_{q^{4}}$ with maximum kernel. In the following table the $q$-degree will be denoted by $k$.

\begin{tabular}{|c|c|c|}
\hline$k$ & polynomial form & conditions \\
\hline 3 & $\operatorname{Tr}(\lambda x)$ & $\lambda \in \mathbb{F}_{q^{4}}^{*}$ \\
\hline 2 & $a_{0} x-x^{q^{2}}$ & $\mathrm{~N}_{q^{4}} / q^{2}\left(a_{0}\right)=1$ \\
\hline 2 & $a_{0} x+a_{1} x^{q}-x^{q^{2}}$ & $\left\{\begin{array}{l}\mathrm{N}_{q^{4}}\left(a_{0}\right)=1 \\
a_{1}^{q+1}=a_{0}^{q^{2}+q+1}-a_{0}^{q}\end{array}\right.$ \\
\hline 1 & $a_{0} x-x^{q}$ & $\mathrm{~N}_{q^{4} / q}\left(a_{0}\right)=1$ \\
\hline
\end{tabular}

Table 1: Linearized polynomials of $\mathbb{F}_{q^{4}}$ with maximum kernel with $s=1$ 


\subsection{The $n=5$ case}

In this section we determine the linearized polynomials over $\mathbb{F}_{q^{5}}$ with maximum kernel. Without loss of generality, we can suppose that the leading coefficient of the polynomial is -1 . Because of Proposition 3.8, we can assume $s \in\{1,2\}$. Corollaries 3.4 and 3.5 cover the cases when the $q^{s}$-degree of $f$ is 1 or 4 . First we suppose that $f$ has $q^{s}$-degree 3 , i.e.

$$
f(x)=a_{0} x+a_{1} x^{q^{s}}+a_{2} x^{q^{2 s}}-x^{q^{3 s}} .
$$

From $(12), f(x)$ has maximum kernel if and only if $a_{0}, a_{1}$ and $a_{2}$ satisfy the following system:

$$
\left\{\begin{array}{l}
a_{1}=-a_{0}^{q^{s}+1} a_{2}^{q^{2 s}} \\
-a_{0}^{q^{3 s}+q^{2 s}+1} a_{2}^{q^{4 s}}+a_{2}^{q^{2 s}+q^{s}} a_{0}=1, \\
a_{2}=-a_{0}^{q^{2 s}+1}+a_{2}^{q^{3 s}+q^{2 s}} a_{0}^{q^{2 s}+q^{s}+1}
\end{array}\right.
$$

which is equivalent to

$$
\left\{\begin{array}{l}
\mathrm{N}\left(a_{0}\right)=1, \\
a_{1}=-a_{0}^{q^{s}+1} a_{2}^{q^{2 s}}, \\
-a_{0}^{q^{3 s}+q^{2 s}+1} a_{2}^{q^{4 s}}+a_{0} a_{2}^{q^{2 s}+q^{s}}=1,
\end{array}\right.
$$

see (A2) of Section 5 .

Suppose now that the $q^{s}$-degree is 2 , i.e.

$$
f(x)=a_{0} x+a_{1} x^{q^{s}}-x^{q^{2 s}} .
$$

By Theorem 3.3 the polynomial $f(x)$ has maximum kernel if and only if its coefficients satisfy

$$
\left\{\begin{array}{l}
a_{0}\left(a_{0}^{q^{2 s}} a_{1}^{q^{3 s}}+a_{1}^{q^{s}}\left(a_{0}^{q^{3 s}}+a_{1}^{q^{3 s}+q^{2 s}}\right)\right)=1, \\
a_{0}^{q^{s}+1}\left(a_{0}^{q^{3 s}}+a_{1}^{q^{3 s}+q^{2 s}}\right)+a_{1}=0
\end{array}\right.
$$

which is equivalent to

$$
\left\{\begin{array}{l}
\mathrm{N}\left(a_{0}\right)=-1, \\
a_{0}^{q^{s}}+a_{1}^{q^{s}+1}=a_{0}^{q^{2 s}+q^{s}+1} a_{1}^{q^{3 s}}
\end{array}\right.
$$

see (A3) of Section 5

Here we list the $q^{s}$-polynomials, $s \in\{1,2\}$ of $\mathcal{L}_{5, q}$ with maximum kernel, up to a non-zero scalar in $\mathbb{F}_{q^{5}}$. Applying the adjoint operation we can obtain the list of $q^{t}$-polynomials, $t \in\{3,4\}$, over $\mathbb{F}_{q^{5}}$ with maximum kernel. As before, the $q^{s}$-degree is denoted by $k$. 


\begin{tabular}{|c|c|c|}
\hline $\mathrm{k}$ & polynomial form & conditions \\
\hline 4 & $\operatorname{Tr}(\lambda x)$ & $\lambda \in \mathbb{F}_{q^{5}}^{*}$ \\
\hline 3 & $a_{0} x+a_{1} x^{q^{s}}+a_{2} x^{q^{2 s}}-x^{q^{3 s}}$ & $\left\{\begin{array}{l}\mathrm{N}\left(a_{0}\right)=1 \\
a_{1}=-a_{0}^{q^{s}}+1 \\
-a_{0}^{q^{3 s}}+q^{2 s}+1\end{array} a_{2}^{q^{4 s}}+a_{0} a_{2}^{q^{2 s}+q^{s}}=1\right.$ \\
\hline 2 & $a_{0} x+a_{1} x^{q^{s}}-x^{q^{2 s}}$ & $\left\{\begin{array}{l}\mathrm{N}\left(a_{0}\right)=-1 \\
a_{1}^{q^{s}}+1\end{array} a_{0}^{q^{s}}=a_{1}^{q^{3 s}} a_{0}^{q^{2 s}+q^{s}+1}\right.$ \\
\hline 1 & $a_{0} x-x^{q^{s}}$ & $\mathrm{~N}\left(a_{0}\right)=1$ \\
\hline
\end{tabular}

Table 2: Linearized polynomials of $\mathbb{F}_{q^{5}}$ with maximum kernel with $s \in\{1,2\}$

\subsection{The $n=6$ case}

In this section we determine the linearized polynomials over $\mathbb{F}_{q^{6}}$ with maximum kernel. Without loss of generality, we can suppose that the leading coefficient of the polynomial is -1 . Because of Proposition 3.8, we can assume $s=1$. Corollaries 3.4 and 3.5 cover the cases when the $q$-degree of $f$ is 1 or 5 . As before, denote by $k$ the $q^{s}$-degree of $f$.

We first consider the case $k=2$, i.e. $f(x)=a_{0} x+a_{1} x^{q^{s}}-x^{q^{2 s}}$. By Theorem 3.3, $f(x)$ has maximum kernel if and only if the coefficients satisfy

$$
\left\{\begin{array}{l}
\mathrm{N}\left(a_{0}\right)=1 \\
\left(a_{0}^{q}+a_{1}^{q+1}\right)^{q^{3}}=a_{0}^{q^{5}+q^{4}+q^{3}}\left(a_{0}^{q}+a_{1}^{q+1}\right) \\
a_{1}^{q^{4}} a_{0}^{q^{3}}+a_{1}^{q^{2}}\left(a_{0}^{q^{4}}+a_{1}^{q^{4}+q^{3}}\right)=-\frac{a_{1}}{a_{0}^{q+1}}
\end{array}\right.
$$

see (A4) of Section 5 .

If $k=3$, then $f(x)=a_{0} x+a_{1} x^{q^{s}}+a_{2} x^{q^{2 s}}-x^{q^{3 s}}$, and by Theorem 3.3 it has maximum kernel if and only the coefficients fulfill

$$
\left\{\begin{array}{l}
\mathrm{N}\left(a_{0}\right)=1 \\
a_{0}^{q^{3}+q+1}+a_{2}^{q^{3}} a_{1}^{q^{2}} a_{0}^{q+1}-a_{2}^{q} a_{1}=a_{0}^{q}, \\
a_{2}^{q+1}=-a_{0}^{q^{3}+q^{2}+q+1} a_{1}^{q^{4}}-a_{1}^{q}, \\
a_{1}^{q+1}=a_{2} a_{0}^{q}+a_{0}^{q^{2}+q+1} a_{2}^{q^{3}}
\end{array}\right.
$$

see (A5) of Section 5. Note that $a_{1}=0$ if and only if $a_{2}=0$ and in this case we get the trace over $\mathbb{F}_{q^{3}}$. 
Finally, let $k=4$. Then the polynomial $f(x)=a_{0} x+a_{1} x^{q^{s}}+a_{2} x^{q^{2 s}}+$ $a_{3} x^{q^{3 s}}-x^{q^{4 s}}$ has maximum kernel if and only if the coefficients satisfy

$$
\left\{\begin{array}{l}
\mathrm{N}\left(a_{0}\right)=1, \\
a_{0}\left(-a_{0}^{q^{4}+q^{2}}+a_{3}^{q^{5}+q^{4}} a_{0}^{q^{4}+q^{3}+q^{2}}+a_{3}^{q^{2}+q}\right)=1, \\
a_{1}=-a_{0}^{q+1} a_{3}^{q^{2}} \\
a_{2}=-a_{0}^{q^{2}+1}+a_{3}^{q^{3}+q^{2}} a_{0}^{q^{2}+q+1} \\
a_{3}=a_{3}^{q^{4}} a_{0}^{q^{3}+q^{2}+1}+a_{3}^{q^{2}} a_{0}^{q^{3}+q+1}-a_{0}^{q^{3}+q^{2}+q+1} a_{3}^{q^{4}+q^{3}+q^{2}},
\end{array}\right.
$$

see (A6) of Section 5

Here we list the $q$-polynomials of $\mathcal{L}_{6, q}$ with maximum kernel, up to a nonzero scalar in $\mathbb{F}_{q^{6}}^{*}$. Applying the adjoint operation we can obtain the list of $q^{5}$-polynomials over $\mathbb{F}_{q^{6}}$ with maximum kernel. 


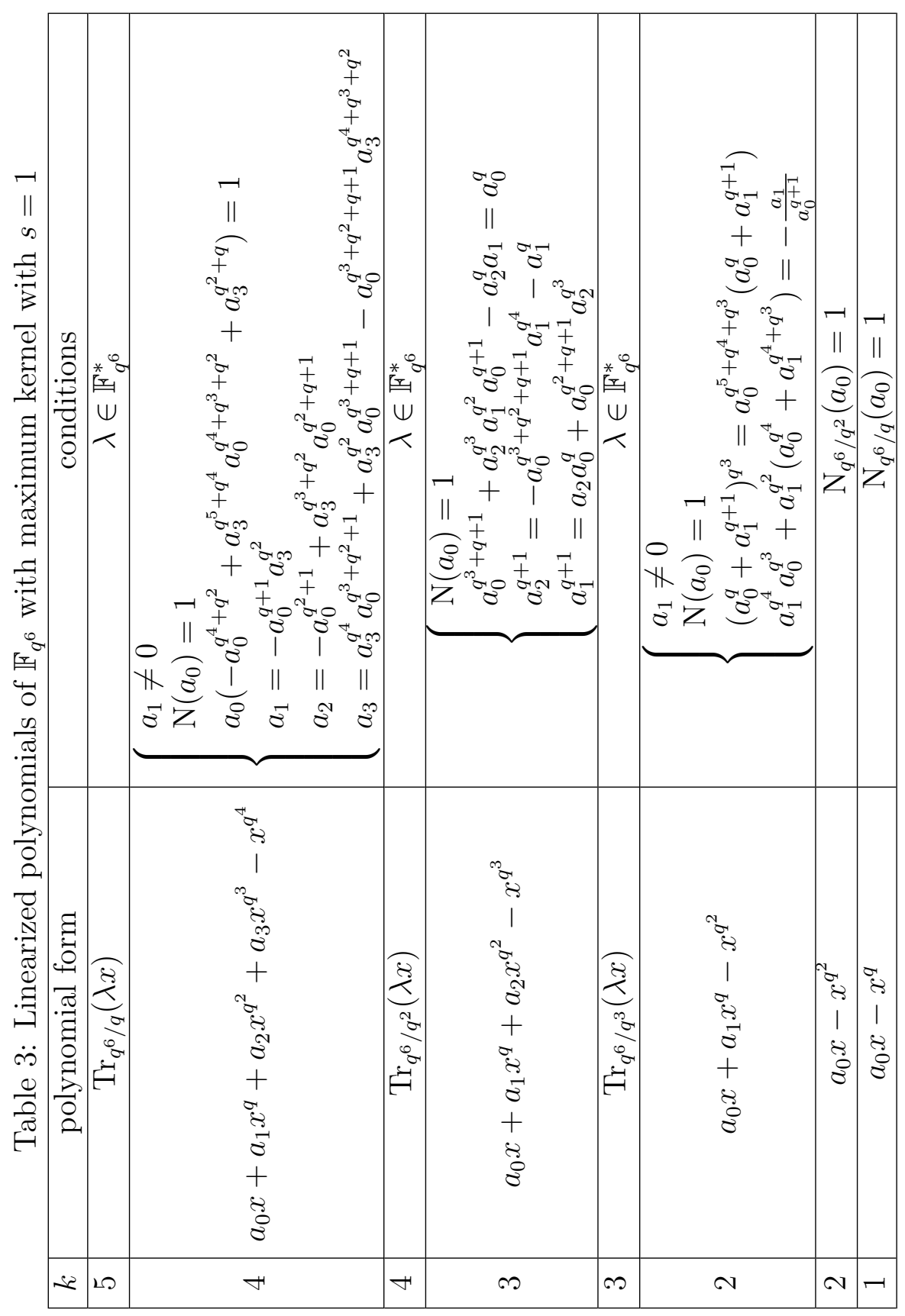




\section{Application}

As an application of Theorem 1.2 we are able to prove the following result on the splitting field of $q$-polynomials.

Theorem 4.1. Let $f(x)=a_{0} x+a_{1} x^{q}+\cdots+a_{k-1} x^{q^{k-1}}-x^{q^{k}} \in \mathbb{F}_{q^{n}}[x]$ with $a_{0} \neq 0$ and let $A$ be defined as in (1). Then the splitting field of $f(x)$ is $\mathbb{F}_{q^{n m}}$ where $m$ is the (multiplicative) order of the matrix $B:=A A^{q} \cdots A^{q^{n-1}}$.

Proof. The derivative of $f(x)$ is non-zero and hence $f(x)$ has $q^{k}$ distinct roots in some algebraic extension of $\mathbb{F}_{q^{n}}$. Suppose that $\mathbb{F}_{q^{n m}}$ is the splitting field of $f(x)$ and let $t$ denote the order of $B$. Then the kernel of the $\mathbb{F}_{q}$-linear $\mathbb{F}_{q^{n m}} \rightarrow \mathbb{F}_{q^{n m}}$ map defined as $x \mapsto f(x)$ has dimension $k$ over $\mathbb{F}_{q}$ and hence by Theorem 1.2 we have

$$
A A^{q} \cdots A^{q^{n m-1}}=I_{k}
$$

Since the coefficients of $A$ are in $\mathbb{F}_{q^{n}}$, this is equivalent to $B^{m}=I_{k}$ and hence $t \mid m$. On the other hand

$$
B^{t}=A A^{q} \cdots A^{q^{n t-1}}=I_{k}
$$

and hence again by Theorem 1.2 the kernel of the $\mathbb{F}_{q^{\text {-linear }}} \mathbb{F}_{q^{n t}} \rightarrow \mathbb{F}_{q^{n t}}$ map defined as $x \mapsto f(x)$ has dimension $k$ over $\mathbb{F}_{q}$. It follows that $\mathbb{F}_{q^{n m}}$ is a subfield of $\mathbb{F}_{q^{n t}}$ from which $m \mid t$.

A further application of Theorem 1.2 is the following.

Theorem 4.2. Let $n, m, s$ and $t$ be positive integers such that $\operatorname{gcd}(s, n m)=$ $\operatorname{gcd}(t, n m)=1$ and $s \equiv t(\bmod m)$. Let $f(x)=a_{0} x+a_{1} x^{q^{s}}+\cdots+$ $a_{k-1} x^{q^{s(k-1)}}-x^{q^{s k}}$ and $g(x)=a_{0} x+a_{1} x^{q^{t}}+\cdots+a_{k-1} x^{q^{t(k-1)}}-x^{q^{t k}}$, where $a_{0}, a_{1}, \ldots, a_{k-1} \in \mathbb{F}_{q^{m}}$. The kernel of $f(x)$ considered as a linear transformation of $\mathbb{F}_{q^{n m}}$ has dimension $k$ if and only if the kernel of $g(x)$ considered as a linear transformation of $\mathbb{F}_{q^{n m}}$ has dimension $k$.

Proof. Denote by $A$ the matrix associated with $f(x)$ as in (1). By hypothesis, $A \in \mathbb{F}_{q^{m}}^{k \times k}$ and it is the same as the matrix associated with $g(x)$. By Theorem 1.2 the kernel of $f(x)$, considered as a linear transformation of $\mathbb{F}_{q^{n m}}$, has dimension $k$ if and only if

$$
A A^{q^{s}} \cdots A^{q^{s(n m-1)}}=I_{k}
$$


Since $s \equiv t(\bmod m)$, we have

$$
A A^{q^{s}} \cdots A^{q^{s(n m-1)}}=A A^{q^{t}} \cdots A^{q^{t(n m-1)}}=I_{k},
$$

and, again by Theorem 1.2. this holds if and only if the kernel of $g(x)$, considered as a linear transformation of $\mathbb{F}_{q^{n m}}$, has dimension $k$.

\section{Addendum}

During the "Combinatorics 2018" conference, the fourth author presented the results of this paper in the talk entitled "On $q$-polynomials with maximum kernel". In the same conference John Sheekey presented a joint work with Gary McGuire [8] in his talk entitled "Ranks of Linearized Polynomials and Roots of Projective Polynomials". It turned out that, independently from the authors of the present paper, they also obtained similar results. 


\section{Appendix}

In this section we develop some calculations regarding the relations on the coefficients of a linearized polynomials with maximum kernel presented in Sections 3.2 , 3.3 and 3.4 , see also [13].

(A1) By Equation (11) with $n=4, s=1$ and $k=2$, we get the conditions

$$
\Sigma:\left\{\begin{array}{l}
a_{0}\left(a_{0}^{q^{2}}+a_{1}^{q^{2}+q}\right)=1 \\
a_{1}=-a_{0}^{q+1} a_{1}^{q^{2}}
\end{array}\right.
$$

By Corollary 3.1, the system $\Sigma$ is equivalent to the following system

$$
\Sigma^{\prime}:\left\{\begin{array}{l}
\mathrm{N}_{q^{4} / q}\left(a_{0}\right)=1, \\
a_{0}\left(a_{0}^{q^{2}}+a_{1}^{q^{2}+q}\right)=1 \\
a_{1}=-a_{0}^{q+1} a_{1}^{q^{2}}
\end{array}\right.
$$

which can be rewritten as follows

$$
\Sigma^{\prime}:\left\{\begin{array}{l}
\mathrm{N}_{q^{4} / q}\left(a_{0}\right)=1, \\
a_{1}^{q^{2}-1}=-\frac{1}{a_{0}^{q+1}}, \\
a_{1}^{q+1}=a_{0}^{q^{2}+q+1}-a_{0}^{q} .
\end{array}\right.
$$

Now consider the system

$$
\Sigma^{*}:\left\{\begin{array}{l}
\mathrm{N}_{q^{4} / q}\left(a_{0}\right)=1, \\
a_{1}^{q+1}=a_{0}^{q^{2}+q+1}-a_{0}^{q} .
\end{array}\right.
$$

Clearly, $S\left(\Sigma^{\prime}\right) \subseteq S\left(\Sigma^{*}\right)$, where $S\left(\Sigma^{\prime}\right)$ and $S\left(\Sigma^{*}\right)$ denote the set of solutions of $\Sigma^{\prime}$ and $\Sigma^{*}$, respectively. Let $\left(a_{0}, a_{1}\right) \in S\left(\Sigma^{*}\right)$, then by using the norm condition on $a_{0}$

$$
\begin{aligned}
& a_{1}^{q^{2}-1}=\left(\frac{1}{a_{0}^{q^{3}}}-a_{0}^{q}\right)^{q-1}=\left(\frac{1-a_{0}^{q+q^{3}}}{a_{0}^{q^{3}}}\right)^{q-1}= \\
& =\frac{1-a_{0}^{1+q^{2}}}{1-a_{0}^{q+q^{3}}} a_{0}^{q^{3}-1}=\frac{1-\frac{1}{a_{0}^{q+q^{3}}}}{1-a_{0}^{q+q^{3}}} a_{0}^{q^{3}-1}=-\frac{1}{a_{0}^{q+1}},
\end{aligned}
$$

i.e. $\left(a_{0}, a_{1}\right) \in S\left(\Sigma^{\prime}\right)$ and hence $S\left(\Sigma^{*}\right)=S\left(\Sigma^{\prime}\right)=S(\Sigma)$. 
(A2) From (11) with $n=5, \operatorname{gcd}(s, 5)=1$ and $k=3$, we get the following conditions:

$$
\Sigma:\left\{\begin{array}{l}
a_{0}\left(a_{1}^{q^{2 s}}+a_{2}^{q^{2 s}+q^{s}}\right)=1, \\
a_{1}=-a_{0}^{q^{s}+1} a_{2}^{q^{2 s}}, \\
a_{2}=-a_{0}^{q^{2 s}+1}-a_{2}^{q^{2 s}} a_{1}^{q^{s}} a_{0} .
\end{array}\right.
$$

By Corollary 3.1, $\Sigma$ is equivalent to

$$
\Sigma^{\prime}:\left\{\begin{array}{l}
\mathrm{N}_{q^{5}} / q\left(a_{0}\right)=1, \\
a_{0}\left(a_{1}^{q^{s}}+a_{2}^{q^{2 s}+q^{s}}\right)=1, \\
a_{1}=-a_{0}^{q^{s}+1} a_{2}^{q^{2 s}}, \\
a_{2}=-a_{0}^{q^{2 s}+1}-a_{2}^{q^{2 s}} a_{1}^{q^{s}} a_{0} .
\end{array}\right.
$$

which can be rewritten as follows

$$
\Sigma^{\prime}:\left\{\begin{array}{l}
\mathrm{N}_{q^{5} / q}\left(a_{0}\right)=1, \\
a_{1}=-a_{0}^{q^{s}+1} a_{2}^{q^{2 s}}, \\
-a_{0}^{q^{3 s}+q^{2 s}+1} a_{2}^{q^{s}}+a_{2}^{q^{2 s}+q^{s}} a_{0}=1, \\
a_{2}=-a_{0}^{q^{2 s}+1}+a_{2}^{q^{3 s}+q^{2 s}} a_{0}^{q^{2 s}+q^{s}+1} .
\end{array}\right.
$$

By raising the third equation to $q^{s}$ and multiplying by $a_{0}^{q^{2 s}+1}$, since $\mathrm{N}\left(a_{0}\right)=1$, we get the fourth equation. Therefore $\Sigma^{\prime}$, and hence $\Sigma$, is equivalent to

$$
\left\{\begin{array}{l}
\mathrm{N}_{q^{5} / q}\left(a_{0}\right)=1, \\
a_{1}=-a_{0}^{q^{s}+1} a_{2}^{q^{2 s}}, \\
-a_{0}^{q^{3 s}+q^{2 s}+1} a_{2}^{q^{4 s}}+a_{0} a_{2}^{q^{2 s}+q^{s}}=1 .
\end{array}\right.
$$

(A3) Applying Theorem 3.3 with $n=5$ and $k=2$, we get that the polynomial $f(x)$ has maximum kernel if and only if its coefficients satisfy

$\Sigma:\left\{\begin{array}{l}Q_{0,5}=a_{0}\left(a_{0}^{q^{2 s}} a_{1}^{q^{3 s}}+a_{1}^{q^{s}}\left(a_{0}^{3^{3 s}}+a_{1}^{q^{3 s}}+q^{2 s}\right)\right)=1, \\ Q_{1,5}=a_{0}^{q^{s}}\left(a_{0}^{q^{3 s}}+a_{1}^{q^{3 s}}+q^{2 s}\right)+a_{1}\left(a_{0}^{q^{2 s}} a_{1}^{q^{3 s}}+a_{1}^{q^{s}}\left(a_{0}^{q^{3 s}}+a_{1}^{q^{3 s}}+q^{2 s}\right)\right)=0,\end{array}\right.$

which is equivalent to

$$
\left\{\begin{array}{l}
\mathrm{N}_{q^{5}} / q\left(a_{0}\right)=-1, \\
a_{0}\left(a_{0}^{q^{2 s}} a_{1}^{q^{3 s}}+a_{1}^{q^{s}}\left(a_{0}^{q^{3 s}}+a_{1}^{q^{3 s}+q^{2 s}}\right)\right)=1, \\
a_{0}^{q^{s}+1}\left(a_{0}^{q^{3 s}}+a_{1}^{q^{3 s}+q^{2 s}}\right)+a_{1}=0,
\end{array}\right.
$$


because of Corollary 3.1 and since $a_{0}^{q^{2 s}} a_{1}^{q^{3 s}}+a_{1}^{q^{s}}\left(a_{0}^{q^{3 s}}+a_{1}^{q^{3 s}}+q^{2 s}\right)=\frac{1}{a_{0}}$. The above system can be rewritten as follows

$$
\left\{\begin{array}{l}
\mathrm{N}_{q^{5} / q}\left(a_{0}\right)=-1 \\
a_{0}^{q^{3 s}}+a_{1}^{q^{3 s}+q^{2 s}}=-\frac{a_{1}}{a_{0}^{q^{s}+1}} \\
a_{1}^{q^{3 s}} a_{0}^{q^{2 s}+q^{s}+1}-a_{1}^{q^{s}+1}=a_{0}^{q^{s}}
\end{array}\right.
$$

which is equivalent to

$$
\left\{\begin{array}{l}
\mathrm{N}_{q^{5} / q}\left(a_{0}\right)=-1 \\
a_{1}^{q^{s}+1}+a_{0}^{q^{s}}=a_{1}^{q^{3 s}} a_{0}^{q^{2 s}+q^{s}+1} \\
a_{1} a_{0}^{q^{4 s}+q^{3 s}+q^{2 s}}=-\frac{a_{1}}{a_{0}^{q^{s}+1}}
\end{array}\right.
$$

If the first and the second equations are satisfied, clearly also the last one is fulfilled, hence $\Sigma$ is equivalent to the following system

$$
\left\{\begin{array}{l}
\mathrm{N}_{q^{5} / q}\left(a_{0}\right)=-1 \\
a_{1}^{q^{s}+1}+a_{0}^{q^{s}}=a_{1}^{q^{3 s}} a_{0}^{q^{2 s}+q^{s}+1}
\end{array}\right.
$$

(A4) By Theorem 3.3, with $n=6, s=1$ and $k=2$, we get

$$
\left\{\begin{array}{l}
Q_{0,6}=a_{0}\left(a_{0}^{q^{2}}\left(a_{0}^{q^{4}}+a_{1}^{q^{4}+q^{3}}\right)+a_{1}^{q}\left(a_{0}^{q^{3}} a_{1}^{q^{4}}+a_{1}^{q^{2}}\left(a_{0}^{q^{4}}+a_{1}^{q^{4}+q^{3}}\right)\right)\right)=1, \\
Q_{1,6}=a_{0}^{q^{2}} a_{1}\left(a_{0}^{q^{4}}+a_{1}^{q^{4}+q^{3}}\right)+\left(a_{1}^{q+1}+a_{0}^{q}\right)\left(a_{0}^{q^{3}} a_{1}^{q^{4}}+a_{1}^{q^{2}}\left(a_{0}^{q^{4}}+a_{1}^{q^{4}+q^{3}}\right)\right)=0,
\end{array}\right.
$$

which is equivalent to

$$
\left\{\begin{array}{l}
a_{0}^{q^{2}}\left(a_{0}^{q^{4}}+a_{1}^{q^{4}+q^{3}}\right)+a_{1}^{q}\left(a_{0}^{q^{3}} a_{1}^{q^{4}}+a_{1}^{q^{2}}\left(a_{0}^{q^{4}}+a_{1}^{q^{4}+q^{3}}\right)\right)=\frac{1}{a_{0}} \\
\frac{a_{1}}{a_{0}}+a_{0}^{q}\left(a_{0}^{q^{3}} a_{1}^{q^{4}}+a_{1}^{q^{2}}\left(a_{0}^{q^{4}}+a_{1}^{q^{4}+q^{3}}\right)\right)=0
\end{array}\right.
$$

i.e.

$$
\left\{\begin{array}{l}
a_{0}^{q^{2}}\left(a_{0}^{q^{4}}+a_{1}^{q^{4}+q^{3}}\right)+a_{1}^{q}\left(a_{0}^{q^{3}} a_{1}^{q^{4}}+a_{1}^{q^{2}}\left(a_{0}^{q^{4}}+a_{1}^{q^{4}+q^{3}}\right)\right)=\frac{1}{a_{0}}, \\
a_{0}^{q^{3}} a_{1}^{q^{4}}+a_{1}^{q^{2}}\left(a_{0}^{q^{4}}+a_{1}^{q^{4}+q^{3}}\right)=-\frac{a_{1}}{a_{0}^{q+1}} .
\end{array}\right.
$$

By Corollary 3.1, the previous system is equivalent to

$$
\left\{\begin{array}{l}
\mathrm{N}\left(a_{0}\right)=1 \\
a_{0}^{q^{2}}\left(a_{0}^{q^{4}}+a_{1}^{q^{4}+q^{3}}\right)+a_{1}^{q}\left(a_{0}^{q^{3}} a_{1}^{q^{4}}+a_{1}^{q^{2}}\left(a_{0}^{q^{4}}+a_{1}^{q^{4}+q^{3}}\right)\right)=\frac{1}{a_{0}} \\
a_{0}^{q^{3}} a_{1}^{q^{4}}+a_{1}^{q^{2}}\left(a_{0}^{q^{4}}+a_{1}^{q^{4}+q^{3}}\right)=-\frac{a_{1}}{a_{0}^{q+1}}
\end{array}\right.
$$


which is equivalent to

$$
\left\{\begin{array}{l}
\mathrm{N}\left(a_{0}\right)=1 \\
a_{0}^{q^{2}}\left(a_{0}^{q}+a_{1}^{q+1}\right)^{q^{3}}-\frac{a_{1}^{q+1}}{a_{0}^{q+1}}=\frac{1}{a_{0}} \\
a_{0}^{q^{3}} a_{1}^{q^{4}}+a_{1}^{q^{2}}\left(a_{0}^{q^{4}}+a_{1}^{q^{4}+q^{3}}\right)=-\frac{a_{1}}{a_{0}^{q+1}},
\end{array}\right.
$$

hence it is equivalent to

$$
\left\{\begin{array}{l}
\mathrm{N}\left(a_{0}\right)=1 \\
\left(a_{0}^{q}+a_{1}^{q+1}\right)^{q^{3}}=a_{0}^{q^{5}+q^{4}+q^{3}}\left(a_{0}^{q}+a_{1}^{q+1}\right), \\
a_{1}^{q^{4}} a_{0}^{q^{3}}+a_{1}^{q^{2}}\left(a_{0}^{q^{4}}+a_{1}^{q^{4}+q^{3}}\right)=-\frac{a_{1}}{a_{0}^{q+1}} .
\end{array}\right.
$$

(A5) By Theorem 3.3 with $n=6, s=1$ and $k=3$, we get

$$
\left\{\begin{array}{l}
a_{0} Q_{2,5}^{q}=1 \\
Q_{0,5}^{q}+a_{1} Q_{2,5}^{q}=0 \\
Q_{1,5}^{q}+a_{2} Q_{2,5}^{q}=0
\end{array}\right.
$$

where

$$
\begin{aligned}
& Q_{0,5}=a_{0}\left(a_{1}^{q^{2}}+a_{2}^{q^{2}+q}\right), \\
& Q_{1,5}=a_{0}^{q} a_{2}^{q^{2}}+a_{1}\left(a_{1}^{q^{2}}+a_{2}^{q^{2}+q}\right), \\
& Q_{2,5}=a_{0}^{q^{2}}+a_{2}^{q^{2}} a_{1}^{q}+a_{2}\left(a_{1}^{q^{2}}+a_{2}^{q^{2}+q}\right),
\end{aligned}
$$

hence we obtain the following system

$$
\left\{\begin{array}{l}
a_{0}\left(a_{0}^{q^{3}}+a_{2}^{q^{3}} a_{1}^{q^{2}}+a_{2}^{q}\left(a_{1}^{q^{3}}+a_{2}^{q^{3}+q^{2}}\right)\right)=1, \\
\frac{a_{1}}{a_{0}}+a_{0}^{q}\left(a_{1}^{q^{3}}+a_{2}^{q^{3}+q^{2}}\right)=0 \\
\frac{a_{2}}{a_{0}}+a_{2}^{q^{3}} a_{0}^{q^{2}}+a_{1}^{q}\left(a_{1}^{q^{3}}+a_{2}^{q^{3}+q^{2}}\right)=0 .
\end{array}\right.
$$

By Corollary 3.1 it is equivalent to

$$
\left\{\begin{array}{l}
\mathrm{N}\left(a_{0}\right)=1, \\
a_{0}\left(a_{0}^{q^{3}}+a_{2}^{q^{3}} a_{1}^{q^{2}}+a_{2}^{q}\left(a_{1}^{q^{3}}+a_{2}^{q^{3}+q^{2}}\right)\right)=1, \\
a_{1}^{q^{3}}+a_{2}^{q^{3}+q^{2}}=-\frac{a_{1}}{a_{0}^{1+q}} \\
\frac{a_{2}}{a_{0}}+a_{2}^{q^{3}} a_{0}^{q^{2}}+a_{1}^{q}\left(a_{1}^{q^{3}}+a_{2}^{q^{3}+q^{2}}\right)=0,
\end{array}\right.
$$


by substituting the third equation into the others we get

$$
\left\{\begin{array}{l}
\mathrm{N}\left(a_{0}\right)=1, \\
a_{0}\left(a_{0}^{q^{3}}+a_{2}^{q^{3}} a_{1}^{q^{2}}-\frac{a_{2}^{q} a_{1}}{a_{0}^{1+q}}\right)=1, \\
a_{1}^{q^{3}}+a_{2}^{q^{3}+q^{2}}=-\frac{a_{1}}{a_{0}^{1+q}}, \\
\frac{a_{2}}{a_{0}}+a_{2}^{q^{3}} a_{0}^{q^{2}}-\frac{a_{1}^{q+1}}{a_{0}^{1+q}}=0,
\end{array}\right.
$$

i.e.

$$
\left\{\begin{array}{l}
\mathrm{N}\left(a_{0}\right)=1 \\
a_{0}^{q^{3}+q+1}+a_{2}^{q^{3}} a_{1}^{q^{2}} a_{0}^{q+1}-a_{2}^{q} a_{1}=a_{0}^{q} \\
a_{2}^{q+1}=-a_{0}^{q^{3}+q^{2}+q+1} a_{1}^{q^{4}}-a_{1}^{q} \\
a_{1}^{q+1}=a_{2} a_{0}^{q}+a_{0}^{q^{2}+q+1} a_{2}^{q^{3}} .
\end{array}\right.
$$

(A6) Equations (11) with $n=6, s=1$ and $k=4$ are

$$
\left\{\begin{array}{l}
a_{0}\left(a_{2}^{q^{2}}+a_{3}^{q^{2}+q}\right)=1 \\
a_{0}^{q} a_{3}^{q^{2}}+a_{1}\left(a_{2}^{q^{2}}+a_{3}^{q^{2}+q}\right)=0 \\
a_{0}^{q^{2}}+a_{3}^{q^{2}} a_{1}^{q}+a_{2}\left(a_{2}^{q^{2}}+a_{3}^{q^{2}+q}\right)=0 \\
a_{1}^{q^{2}}+a_{3}^{q^{2}} a_{2}^{q}+a_{3}\left(a_{2}^{q^{2}}+a_{3}^{q^{2}+q}\right)=0
\end{array}\right.
$$

which, by Corollary 3.1, is equivalent to

$$
\left\{\begin{array}{l}
\mathrm{N}\left(a_{0}\right)=1 \\
a_{0}\left(a_{2}^{q^{2}}+a_{3}^{q^{2}+q}\right)=1 \\
a_{0}^{q} a_{3}^{q^{2}}+a_{1}\left(a_{2}^{q^{2}}+a_{3}^{q^{2}+q}\right)=0 \\
a_{0}^{q^{2}}+a_{3}^{q^{2}} a_{1}^{q}+a_{2}\left(a_{2}^{q^{2}}+a_{3}^{q^{2}+q}\right)=0 \\
a_{1}^{q^{2}}+a_{3}^{q^{2}} a_{2}^{q}+a_{3}\left(a_{2}^{q^{2}}+a_{3}^{q^{2}+q}\right)=0
\end{array}\right.
$$

thus it can be rewritten as follows

$$
\left\{\begin{array}{l}
\mathrm{N}\left(a_{0}\right)=1 \\
a_{2}^{q^{2}}+a_{3}^{q^{2}+q}=\frac{1}{a_{0}} \\
a_{0}^{q} a_{3}^{q^{2}}+\frac{a_{1}}{a_{0}}=0 \\
a_{0}^{q^{2}}+a_{3}^{q^{2}} a_{1}^{q}+\frac{a_{2}}{a_{0}}=0 \\
a_{1}^{q^{2}}+a_{3}^{q^{2}} a_{2}^{q}+\frac{a_{3}}{a_{0}}=0
\end{array}\right.
$$


and hence

$$
\left\{\begin{array}{l}
\mathrm{N}\left(a_{0}\right)=1 \\
a_{0}\left(a_{2}^{q^{2}}+a_{3}^{q^{2}+q}\right)=1 \\
a_{1}=-a_{0}^{q+1} a_{3}^{q^{2}} \\
a_{2}=-a_{0}^{q^{2}+1}-a_{3}^{q^{2}} a_{1}^{q} a_{0} \\
a_{3}=-a_{1}^{q^{2}} a_{0}-a_{3}^{q^{2}} a_{2}^{q} a_{0}
\end{array}\right.
$$

i.e.

$$
\left\{\begin{array}{l}
\mathrm{N}\left(a_{0}\right)=1, \\
a_{0}\left(-a_{0}^{q^{4}+q^{2}}+a_{3}^{q^{5}+q^{4}} a_{0}^{q^{4}+q^{3}+q^{2}}+a_{3}^{q^{2}+q}\right)=1, \\
a_{1}=-a_{0}^{q+1} a_{3}^{q^{2}} \\
a_{2}=-a_{0}^{q^{2}+1}+a_{3}^{q^{3}+q^{2}} a_{0}^{q^{2}+q+1}, \\
a_{3}=a_{3}^{q^{4}} a_{0}^{q^{3}+q^{2}+1}+a_{3}^{q^{2}} a_{0}^{q^{3}+q+1}-a_{0}^{q^{3}+q^{2}+q+1} a_{3}^{q^{4}+q^{3}+q^{2}} .
\end{array}\right.
$$




\section{References}

[1] D. Bartoli, M. Giulietti, G. Marino and O. Polverino: Maximum scattered linear sets and complete caps in Galois spaces, Combinatorica 38(2) (2018), 255-278.

[2] B. Csajbók, G. Marino and O. Polverino: Classes and equivalence of linear sets in $\mathrm{PG}\left(1, q^{n}\right)$, J. Combin. Theory Ser. A 157 (2018), 402-426.

[3] P. Delsarte: Bilinear forms over a finite field, with applications to coding theory, J. Combin. Theory Ser. A 25 (1978), 226-241.

[4] U. Dempwolff, J. C. Fisher and A. Herman: Semilinear transformations over finite fields are Frobenius maps, Glasg. Math. J. 42.2 (2000): 289-295.

[5] E. Gabidulin: Theory of codes with maximum rank distance, Problems of information transmission, 21(3) (1985), 3-16.

[6] R. Gow and R. Quinlan: Galois theory and linear algebra, Linear Algebra Appl. 430 (2009), 1778-1789.

[7] R. Gow and R. Quinlan: Galois extensions and subspaces of alterning bilinear forms with special rank properties, Linear Algebra Appl. 430 (2009), 2212-2224.

[8] G. McGuire And J. Sheekey: A Characterization of the Number of Roots of Linearized and Projective Polynomials in the Field of Coefficients, https://arxiv.org/abs/1806.05853.

[9] S. Lang: Algebraic groups over finite fields, Amer. J. Math. 78 (1956), 555-563.

[10] R. Lidl and H. Niederreiter: Finite fields, Cambridge university press, Vol. 20, 1997.

[11] O. Ore: On a special class of polynomials, Trans. Amer. Math. Soc. 35 (1933), 559-584.

[12] J. Sheekey: A new family of linear maximum rank distance codes, Adv. Math. Commun. 10(3) (2016), 475-488. 
[13] F. ZulLo: Linear codes and Galois geometries: between two worlds, PhD thesis, Università degli Studi della Campania "Luigi Vanvitelli" (2018).

Bence Csajbók

MTA-ELTE Geometric and Algebraic Combinatorics Research Group

ELTE Eötvös Loránd University, Budapest, Hungary

Department of Geometry

1117 Budapest, Pázmány P. stny. 1/C, Hungary

csajbokb@cs.elte.hu

Giuseppe Marino

Dipartimento di Matematica e Fisica, Università degli Studi della Campania "Luigi Vanvitelli", Viale Lincoln 5, I- 81100 Caserta, Italy

Dipartimento di Matematica e Applicazioni "Renato Caccioppoli" Università degli Studi di Napoli "Federico II", Via Cintia, Monte S.Angelo I-80126 Napoli, Italy

giuseppe.marino@unicampania.it,giuseppe.marino@unina.it

Olga Polverino and Ferdinando Zullo

Dipartimento di Matematica e Fisica, Università degli Studi della Campania "Luigi Vanvitelli", I- 81100 Caserta, Italy

olga.polverino@unicampania.it, ferdinando.zullo@unicampania.it 